\title{
Stability Assessment of OCO-2 Radiometric Calibration Using Aqua MODIS as a Reference
}

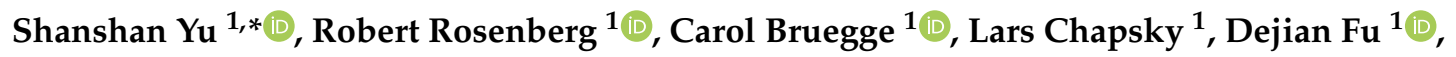 \\ Richard Lee ${ }^{1}$, Thomas Taylor ${ }^{2}$, Heather Cronk ${ }^{2}$, Christopher O'Dell ${ }^{2}$, Amit Angal ${ }^{3} \mathbb{C D}_{\text {, }}$ \\ Xiaoxiong Xiong ${ }^{4}$, David Crisp ${ }^{1}\left(\mathbb{D}\right.$ and Annmarie Eldering ${ }^{1(D)}$ \\ 1 Jet Propulsion Laboratory, California Institute of Technology, Pasadena, CA 91109, USA; \\ rob.rosenberg@jpl.nasa.gov (R.R.); carol.j.bruegge@jpl.nasa.gov (C.B.); lars.chapsky@jpl.nasa.gov (L.C.); \\ dejian.fu@jpl.nasa.gov (D.F.); Richard.A.Lee@jpl.nasa.gov (R.L.); david.crisp@jpl.nasa.gov (D.C.); \\ Annmarie.Eldering@jpl.nasa.gov (A.E.) \\ 2 Cooperative Institute for Research in the Atmosphere, Fort Collins, CO 80521, USA; \\ tommy.taylor@colostate.edu (T.T.); Heather.Cronk@colostate.edu (H.C.); Christopher.ODell@colostate.edu (C.O.) \\ 3 Science Systems and Applications, Inc., Lanham, MD 20706, USA; amit.angal@ssaihq.com \\ 4 Sciences and Exploration Directorate, NASA Goddard Space Flight Center, Greenbelt, MD 20706, USA; \\ Xiaoxiong.Xiong-1@nasa.gov \\ * Correspondence: Shanshan.Yu@jpl.nasa.gov; Tel.: +1-818-354-5829
}

Received: 4 March 2020; Accepted: 12 April 2020; Published: 17 April 2020

\begin{abstract}
With three imaging grating spectrometers, the Orbiting Carbon Observatory-2 (OCO-2) measures high spectral resolution spectra $(\lambda / \Delta \lambda \approx 19,000)$ of reflected solar radiation within the molecular oxygen $\left(\mathrm{O}_{2}\right)$ A-band at $0.765 \mu \mathrm{m}$ and two carbon dioxide $\left(\mathrm{CO}_{2}\right)$ bands at 1.61 and $2.06 \mu \mathrm{m}$. OCO-2 uses onboard lamps with a reflective diffuser, solar observations through a transmissive diffuser, lunar measurements, and surface targets for radiometric calibration and validation. Separating calibrator aging from instrument degradation poses a challenge to OCO-2. Here we present a methodology for trending the OCO-2 Build $8 \mathrm{R}$ radiometric calibration using OCO-2 nadir observations over eight desert sites and nearly simultaneous observations from Moderate Resolution Imaging Spectroradiometer (MODIS) with sensor viewing zenith angles of $15 \pm 0.5^{\circ}$. For the $\mathrm{O}_{2} \mathrm{~A}$-band, this methodology is able to quantify a drift of $-0.8 \pm 0.1 \%$ per year and capture a small error in correcting the aging of the solar calibrator. For the other two OCO-2 bands, no measurable changes were seen, indicating less than $0.1 \%$ and less than $0.3 \%$ per year drift in the radiometric calibration of Band 2 and Band 3, respectively.
\end{abstract}

Keywords: radiometric calibration; validation; OCO-2; MODIS; pseudo invariant calibration sites

\section{Introduction}

The Orbiting Carbon Observatory 2 (OCO-2) satellite launched in 2014 and the Orbiting Carbon Observatory 3 (OCO-3) payload launched in 2019 are NASA Earth science missions designed to return estimates of $\mathrm{CO}_{2}$ with the precision, accuracy, resolution and coverage needed to identify sources and sinks of $\mathrm{CO}_{2}$ on regional scales [1-4]. OCO-2 and OCO-3 use high resolution imaging spectrometers to measure solar radiance reflected by the Earth atmosphere and surface at high spectral resolution $(\lambda / \Delta \lambda \approx 19,000)$. After calibration, these measurements are analyzed to estimate the column-average dry air mole fraction of $\mathrm{CO}_{2}$ (XCO2) in the atmosphere [1]. The daily variance of XCO2 at regional scales is on the order of $1 \mathrm{ppm}$ while the background is around $400 \mathrm{ppm}$. To achieve the required $0.25 \%$ accuracy in the retrieved XCO2 estimations, the OCO missions require a $5 \%$ accuracy at $1 \sigma$ confidence level on absolute radiometric calibration. The OCO-2 instruments were characterized and 
calibrated during preflight testing [5], but the radiometric performance must be tracked on orbit as various components degrade.

The OCO-2 and OCO-3 missions use three grating spectrometers to measure reflected sunlight within the $\mathrm{O}_{2} \mathrm{~A}$-band at $0.765 \mu \mathrm{m}$ (hereafter Band 1 or $\mathrm{ABO} 2$ ) and two $\mathrm{CO}_{2}$ bands at $1.61 \mu \mathrm{m}$ (hereafter Band 2 or WCO2) and $2.06 \mu \mathrm{m}$ (hereafter Band 3 or SCO2). The three spectrometers share common entrance optics, and each band's spectrum is recorded on a unique Focal Plane Array (FPA) detector. One dimension of the FPA records the spectrum, which is dispersed across its 1016 active columns. The other dimension of the FPA records angular variations along the $\sim 1$-degree wide spectrometer slit. The middle 0.8-degree wide region illuminated by the slit is recorded by 160 rows of the FPA, which are binned into eight, $\sim 20$-pixel wide spatial footprints. The eight, along-slit spectra are recorded at $0.333 \mathrm{~s}$ intervals, yield surface footprints that are $\sim 1.29 \mathrm{~km} \times 2.25 \mathrm{~km}$ when the boresight is pointed at the local nadir and the slits are oriented perpendicular to the ground track. We refer the readers to Ref. [1] for more details on how the grating spectrometer returns high resolution $\mathrm{O}_{2}$ and $\mathrm{CO}_{2}$ spectra.

Various methodologies presented in Table 1 are used for OCO-2 radiance calibration and validation. We refer the readers to Ref. [6] for detailed descriptions of the strengths and weaknesses of each methodology. The latest OCO-2 data version, Build 8R, uses solar and lunar observations and onboard lamp and dark observations for radiometric calibration [1]. The absolute radiometric calibration is monitored by vicarious calibration at Railroad Valley (RRV), Nevada, USA [6]. The lunar calibration measurements are typically performed using the $3 / 4$ waxing gibbous phase andtThese observations were also taken near full phase before July 2019. These lunar measurements are used to trend the degradation of the solar diffuser and to identify and correct long-term trends in the throughput of the spectrometers in the current, $8 \mathrm{R}$ data product and in the updated 10R data product that is currently in production and will be released in late 2020.

This paper assesses the OCO-2 radiometric calibration stability using OCO-2 nadir observations over Pseudo Invariant Calibration Sites (PICS) and nearly simultaneous MODIS observations. Changes in the OCO-2/MODIS radiance ratio with time are used to quantify the drift of OCO-2 Build 8R. Cross calibration over PICS is expected to play an important role in the radiometric calibration of OCO-3, which is equipped with onboard lamps, but will take very limited lunar measurements and no solar measurements due to its configuration aboard the International Space Station.

Table 1. OCO-2 and OCO-3 inflight radiometric calibration/validation methodologies and frequencies.

\begin{tabular}{ccc}
\hline Calibration Type & OCO-2 Frequency & OCO-3 Frequency \\
\hline Vicarious at RRV & $1 \times$ per month & $5 \times$ per 3 months \\
Solar calibration & $10 \times$ per day & Not possible \\
Lamp calibration & $10 \times$ per day & $15 \times$ per day \\
Lunar calibration & 1 or $2 \times$ per lunar cycle & $4-6 \times$ per year (goal) \\
Cross calibration over PICS & 1 chance per site every 16 days & Varies \\
\hline
\end{tabular}

\section{Materials and Methods}

\subsection{Sensor Background and Data Products Used in this Study}

Table 2 lists the OCO-2 and Aqua MODIS sensor characteristics and data products used for this study. OCO-2 was inserted at the front of the $705 \mathrm{~km}$ Afternoon Constellation (A-Train) on 3 August 2014, and flies about $7 \mathrm{~min}$ ahead of the Aqua spacecraft. The OCO-2 spectrometer collected its first light spectra on 6 August 2014, and has been returning science data since 6 September 2014 . OCO-2 has eight along-slit footprints with dimensions of $\sim 2.25 \mathrm{~km}$ along track and $1.3 \mathrm{~km}$ cross track at nadir, with a total swath width of $10 \mathrm{~km}$. Data structures in OCO-2 product files are indexed by sounding, where a sounding is the co-located spectra from the three spectrometers at a location (footprint). The sounding_id variable is the primary identifier that allows data to be linked between different processing levels. The sounding_id is a composite of the time the data was acquired and the 
footprint number (f) in the form yyyymmddhhmmsssf. OCO-2 acquires eight soundings every $0.333 \mathrm{~s}$, each contains three spectra at $0.765,1.61$, and $2.06 \mu \mathrm{m}$.

The MODIS instrument has stringent performance requirements that are accomplished by a set of on-board calibrators that include a solar diffuser (SD) with its on-orbit degradation tracked using a solar diffuser stability monitor (SDSM). Because of its on-orbit calibration capability and data quality, the MODIS instrument has been used as a calibration and/or a validation reference by many Earth observing sensors. The Global Space-based Inter-Calibration System (GSICS), an international collaborative effort to harmonize the quality of observations from operational weather and environmental satellites, uses Aqua MODIS as its calibration reference sensor in the reflective solar spectral region [7].

In comparison with Terra MODIS, Aqua MODIS has experienced far less degradation of its optics and on-board calibrators resulting in an overall better performance. The on-orbit calibration of Aqua MODIS is regularly updated via offline look-up-tables that are derived using a combination of solar and lunar measurements. The on-orbit uncertainty for Aqua MODIS reflective solar bands continues to be stable over the mission and below the $2 \%$ requirement in reflectance and $5 \%$ in radiance $[8,9]$.

Table 2. Sensor characteristics and data used in this study.

\begin{tabular}{lll}
\hline & OCO-2 & Aqua MODIS \\
\hline Launched & 2-July-2014 & 4-May-2002 \\
Equator crossing time & $1: 36 \mathrm{PM}$ (local) & $1: 37 \mathrm{PM}$ (local) \\
Altitude & $705 \mathrm{~km}$ & $705 \mathrm{~km}$ \\
Swath width & $<10 \mathrm{~km}$ at nadir & $2330 \mathrm{~km}$ \\
Spatial resolution & $1.29 \mathrm{~km} \times 2.25 \mathrm{~km}$ at nadir & $250-500 \mathrm{~m}$ \\
\hline Bands $(\mu \mathrm{m})$ & B1 $0.7576-0.7726$ & B1 $0.620-0.670$ \\
& & B2 0.841-0.876 \\
& B2 $1.5906-1.6218$ & B6 1.628-1.652 \\
& B3 2.0431-2.0834 & B7 2.105-2.155 \\
\hline Data versions & B8R & C6.1 \\
\hline Data products & L2ABP (cloud screening) & MYD06 (cloud screening) \\
& L2IDP (cloud screening) & MYD02HKM (radiance) \\
& L2Dia (radiance) & \\
\hline
\end{tabular}

Examples of OCO-2 spectra are shown in Figure 1: the X-axis, wavelength, represents the 1016 spectral channels and the Y-axis is spectral radiance. The horizontal black lines illustrate the so-called spectral continuum level, which is estimated as the average of the ten largest radiance values. A single continuum level is assigned to each spectrum, although the true continuum varies slightly with wavelength. These are stored as three variables in the Level 2 Diagnostic (L2Dia) product, signal_o2_fph, signal_weak_co2_fph, and signal_strong_co2_fph. The OCO-2 Band 1 at $0.765 \mu \mathrm{m}$ includes several $\mathrm{L} 1 \mathrm{~b}$ columns that are free from atmospheric gaseous absorption features. The estimate represents the true continuum of this band well. Band 2 at $1.61 \mu \mathrm{m}$ contains fewer such columns and its estimate should be taken as a rough approximation of the true continuum. Band 3 at $2.06 \mu \mathrm{m}$ contains no columns that are free from atmospheric gaseous absorption features. Therefore the estimated Band 3 continuum level varies with the atmospheric $\mathrm{CO}_{2}$ column abundance.

In the present study, the OCO-2 continuum for each band is compared to the in-band spectral radiance of the matching MODIS band. As shown in Figure 2, the OCO-2 Band 1 lies between MODIS Bands 1 and 2, and is therefore compared to their average; OCO-2 Bands 2 and 3 are compared to MODIS Band 6 and Band 7, respectively. Figure 2 also shows the relative spectral response (RSR) functions for the four relevant MODIS Bands (see the expanded view in the bottom panels). OCO-2 disperses light within each of its three bands onto 1016 spectral channels. The instrument line shape (ILS) functions, which describe the relative spectral response of each channel, have been measured to $0.2 \%$ in preflight testing [1], and have not changed by measurable amounts on-orbit [10]. The OCO-2 ILS functions are not shown in Figure 2 because their $<0.1 \mathrm{~nm}$ widths cannot be resolved at this spectral 
resolution: the continuum is spectrally flat across the integral of $\Delta \lambda<0.1 \mathrm{~nm}$ for each channel and can be pulled out when theoretically deriving the observed OCO-2 radiance at the continuum

$$
L=\int L_{\lambda} I L S_{\lambda} \mathrm{d} \lambda / \int I L S_{\lambda} \mathrm{d} \lambda=L_{\lambda} \int I L S_{\lambda} \mathrm{d} \lambda / \int I L S_{\lambda} \mathrm{d} \lambda=L_{\lambda}
$$

where $\mathrm{L}_{\lambda}$ denotes the top of atmosphere (TOA) radiance profile; ILS $_{\lambda}$ is the instrument line shape function of OCO-2.

(a)

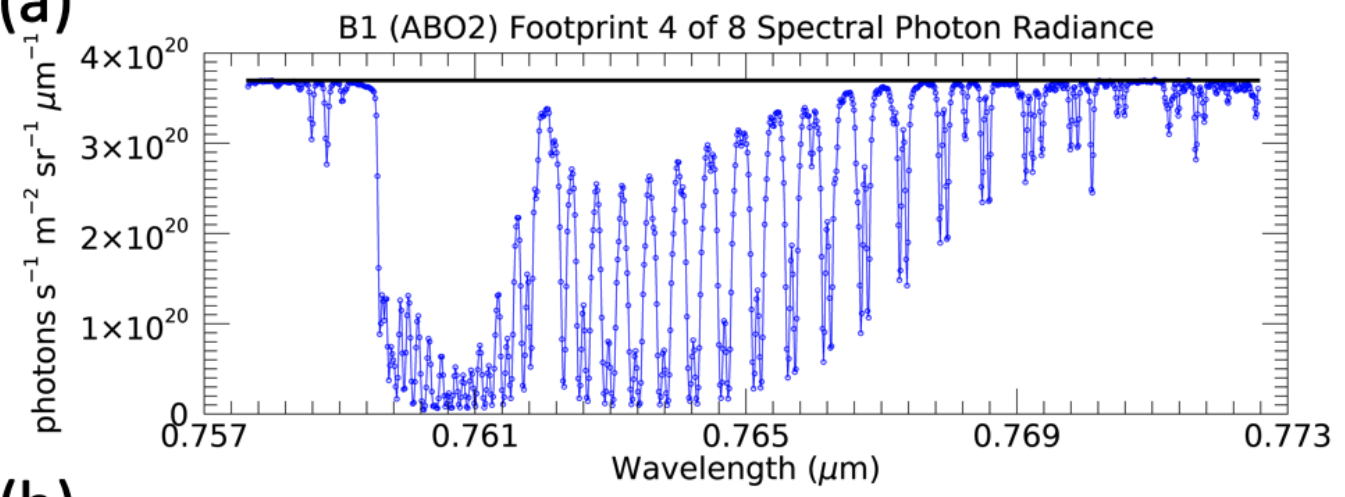

(b)

Wavelength $(\mu \mathrm{m})$

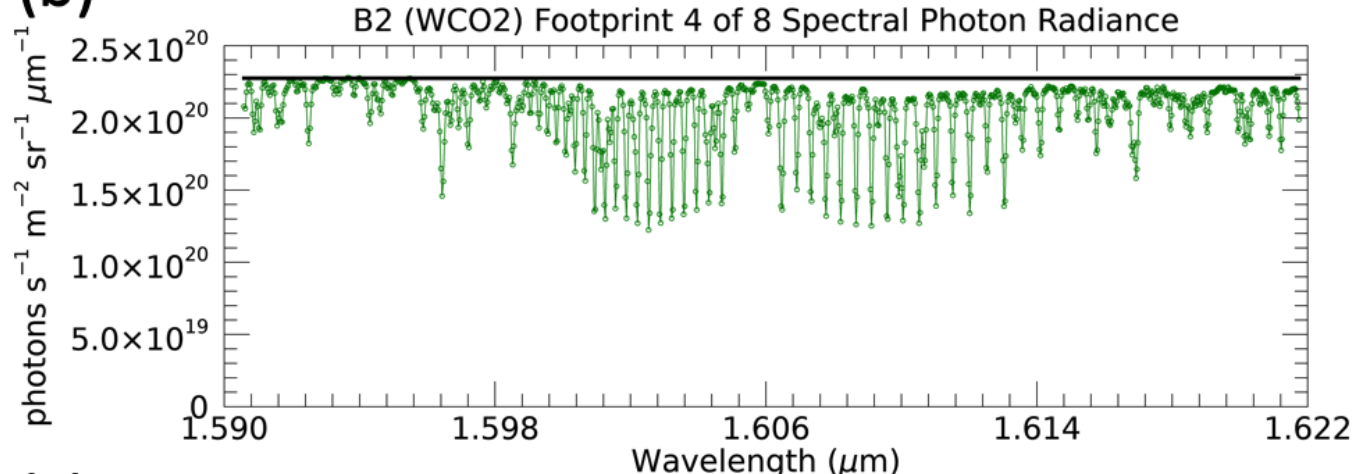

(c)

Wavelength $(\mu \mathrm{m})$

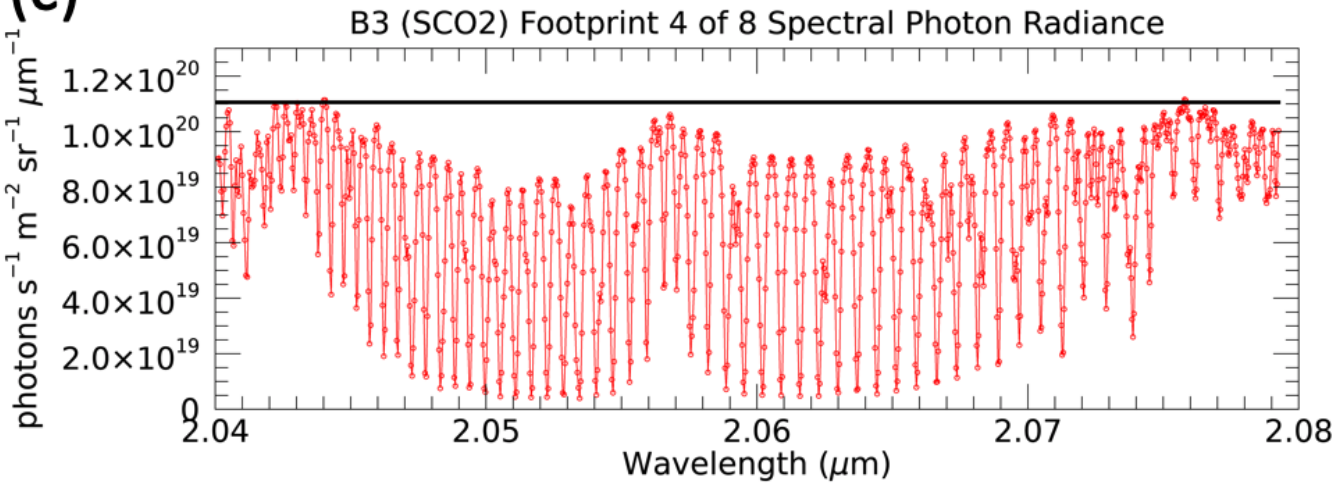

Figure 1. Examples of OCO-2 spectra taken over Egypt1 (sounding_id=2019071311331034, orbit \#26754): Panel (a) for Band 1; Panel (b) for Band 2; Panel (c) for Band 3. The grating of each band disperses light onto 1016 spectral channels of a focal plane array, yielding three spectra with a resolution of $\Delta \lambda \approx 0.04$, 0.08 , and $0.1 \mathrm{~nm}$, respectively. The black horizontal line is the average of the 10 largest radiance values, which is used to estimate the continuum signal. This OCO-2 continuum estimate is compared to the spectral radiance of the matching MODIS band. 
Note that OCO-2 only measures one linear polarization while MODIS is a polarization insensitive instrument and measures the total intensity. If the incident light were unpolarized, the OCO-2 radiance would be half of the MODIS radiance. If the incident light were polarized in the direction where OCO-2 is most responsive, then OCO2 would measure all the incident light and the OCO-2 radiance would be the same as the MODIS radiance. If the incident light were polarized perpendicular to OCO-2's responsive direction, then no light would be measured by OCO-2. While sunlight incident at the top of Earth's atmosphere is not polarized, scattering by the atmosphere and surface can introduce $\sim 1 \%$ polarization in the reflected sunlight observed by OCO-2 over desert scenes. In the present study, we assume that the reflected sunlight observed by OCO-2 is unpolarized and multiply the OCO-2 radiance by a factor of 2 and then compare it to the MODIS counterpart. This approximation may contribute to the seasonal oscillation seen in OCO-2/MODIS radiance ratio trending (see Section 4 for more details), and may need to be addressed when this method is expanded to include absolute calibration

Note that small spectral shifts of OCO-2, on the order of $\sim 0.08$ of a pixel, have been observed in-flight, and routinely monitored and corrected in orbit using measurements of the positions and shapes of solar lines observed through the solar diffuser [1]. Even without corrections, these incredibly small spectral drifts of OCO-2 have no impact on the current study as the OCO-2 continuum radiance is estimated as the average of the 10 largest radiance values in a spectrum, and thus is free of small wavelength calibration errors.

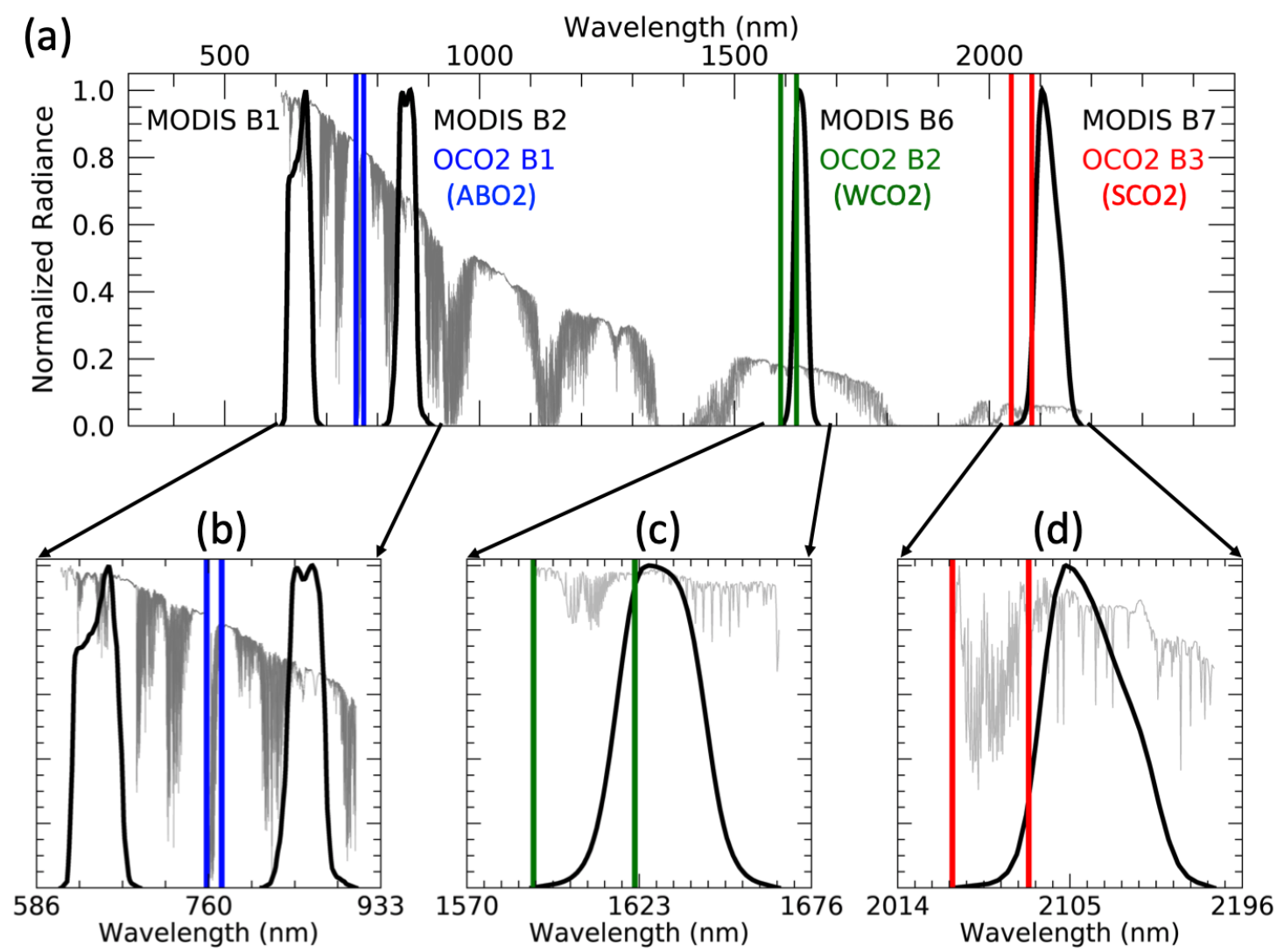

Figure 2. Wavelength ranges measured by OCO-2 overlaid with the relative spectral response functions of MODIS Bands 1, 2, 6 and 7, along with Top of Atmosphere radiance simulated with MODTRAN: Panel (a) for overview from 500-2300 nm,; Panel (b) for expanded view around $760 \mathrm{~nm}$; Panel (c) for expanded view around $1623 \mathrm{~nm}$; Panel (d) for expanded view around $2105 \mathrm{~nm}$. 


\subsection{Pics Used in this Study}

Table 3 lists the eight PICS used for this study. We first searched for OCO-2 clear-sky nadir observations over the twenty original PICS that were well characterized by Refs. [11-13]. However, due to the narrow OCO-2 swath of $<10 \mathrm{~km}$, repeating ground track, and resulting spatial coverage gaps, OCO-2 only has nadir observations over the eight PICS listed in Table 3 and has no nadir observations over the other twelve PICS. Note that two of the eight PICS, Libya1 and Mauritania1 (boldface in Table 3), are recommended by Committee on Earth Observation Satellites Infrared and Visible Optical Sensors Subgroup (CEOS IVOS) as cross calibration reference sites. The other four PICS recommended by CEOS IVOS, Algeria3, Libya4, Mauritania2, and Algeria5, are not included in the current study due to lack of OCO-2 nadir observations over them.

Table 3. PICS used in this study (Boldfaced are two sites recommended by CEOS IVOS).

\begin{tabular}{lcc}
\hline Site Name & Latitude $\left(^{\circ}\right)$ & Longitude $\left(^{\circ}\right)$ \\
\hline Arabia1 & 18.88 & 46.76 \\
Algeria1 & 23.65 & -0.55 \\
Algeria2 & 26.00 & -1.20 \\
Algeria4 & 30.10 & 5.70 \\
Egypt1 & 27.12 & 26.10 \\
Libya1 & $\mathbf{2 4 . 4 2}$ & $\mathbf{1 3 . 3 5}$ \\
Libya2 & 25.05 & 20.48 \\
Mauritania1 & $\mathbf{1 9 . 4 0}$ & $-\mathbf{9 . 3 0}$ \\
\hline
\end{tabular}

\subsection{Inter-Comparison Data Processing}

This study quantifies the time dependence of OCO-2/MODIS radiance ratio to assess the OCO-2 radiometric calibration stability. The data products used include Level 2 A-band Pre-processor (L2ABP), Level 2 Iterative maximum a posteriori differential optical absorption spectroscopy (IMAP-DOAS) Pre-processor (L2IDP), and Level 2 Diagnostic (L2Diag) products generated by the Build 8R algorithm, and the OCO-2 Collocation products for the MODIS / Aqua Calibrated Radiances 5-Min L1B Swath (OCO-2/MYD02HKM) and MODIS Aqua 1-km Cloud Product (OCO-2/MYD061K) from OCO-2/MODIS Collocation Products, Version 3, June 2018 [14]. The OCO-2 and MODIS radiances are from L2Dia and OCO-2/MYD02HKM, respectively. The three other products are used for cloud screening. OCO-2 data products are available at https:/ / disc.gsfc.nasa.gov/information/data-release? title=OCO-2\%20Version\%208. OCO-2/MODIS Collocation Products are produced by the Cooperative Institute for Research in the Atmosphere (CIRA) Data Processing Center (DPC) at Colorado State University in collaboration with the A-Train Data Depot (ATDD) at the Goddard Earth Sciences Data and Information Services Center (GES-DISC). We refer the readers to Ref. [15] for details on the collocation methodology. The OCO-2/MODIS collocated data products are available upon request. Briefly, the OCO-2/MODIS collocated data products are produced by:

(1) Identifying MODIS 5-min granules corresponding to a single OCO-2 L1B science granule using OCO-2 time (typically nine MODIS 5-min granule per OCO-2 granule);

(2) Aggregating information from all nine 5-min MODIS granules into a single file and performing a pixel-by-pixel match to the OCO-2 sounding locations (a matched MODIS pixel is the one whose center location is closest to the center location of a OCO-2 sounding);

(3) Writing an output file containing MODIS geolocation and science data within $\pm 50 \mathrm{~km}$ of the OCO-2 ground target, OCO-2 geolocation and sounding IDs, the MODIS pixel indices in original MODIS file for each match-up, and the distance between matched pixels. OCO-2/MYD02HKM is the half-kilometer radiance product containing $500 \mathrm{~m}$ and aggregated $250 \mathrm{~m}$ Earth View MODIS data that can be used to calculate radiance; The OCO-2 and interpolated half-kilometer MODIS geolocation coordinates are provided as well as origin file information and the MODIS pixel indices for each pixel match-up and the distance between matched pixels. OCO-2/MYD061KM is the 1-km cloud product 
containing selected 1-km MODIS cloud, satellite, and scene properties as well as the OCO-2 and 1-km MODIS geolocation, origin file information, and the MODIS pixel indices for each pixel match-up and the distance between matched pixels.

Figure 3 shows the schematic flow diagram for our inter-comparison data processing for each of the eight sites, which can be detailed in the following steps:

(1) Defining a Region Of Interest as a $0.9^{\circ} \times 0.9^{\circ}$ latitude by longitude area around the site center.

(2) Extracting OCO-2 soundings observed in nadir and over the ROI, from L2ABP, L2IDP and L2Dia. Converting radiances from OCO-2 units (photons s${ }^{-1} \mathrm{~m}^{-2} \mathrm{sr}^{-1} \mu \mathrm{m}^{-1}$ ) to MODIS units $\left(\mathrm{W} \mathrm{m} \mathrm{m}^{-2} \mathrm{sr}^{-1} \mu \mathrm{m}^{-1}\right)$ using

$$
L \_ \text {in_MODIS_units }=L \_ \text {in_OCO2_units } \times 1.98645 \times 10^{-19} / \lambda
$$

where $\lambda$ equals $0.765,1.605$ and $2.06 \mu \mathrm{m}$, respectively. Note here the unit conversion uses

$$
\begin{aligned}
\text { photon_energy } & =h c / \lambda \\
& =1.23984193 \mathrm{eV} \cdot \mu \mathrm{m} / \lambda \text { in } \mu \mathrm{m} \\
& =1.23984193 \mathrm{eV} \frac{1.60217733 \times 10^{-19} \mathrm{~W} \cdot \mathrm{s}}{1 \mathrm{eV}} / \lambda \\
& =1.98645 \times 10^{-19} \mathrm{~W} \cdot \mathrm{s} / \lambda
\end{aligned}
$$

(3) Mapping MODIS 1-km cloud data to OCO-2 sounding-by-sounding: (i) for each OCO-2 sounding, the closest MODIS pixel is located using the matched MODIS pixel indices stored in OCO-2/MYD061KM; (ii) MODIS cloud mask data are extracted for pixels inside a matchup box of $7 \times 7$ MODIS pixels centered at the closest MODIS pixel and less than $2 \mathrm{~km}$ away from the center of the OCO-2 sounding; (iii) a final clear flag is assigned if only all MODIS cloud mask data obtained in Step (ii) are less than 2, indicating clear or probably clear.

(4) Mapping MODIS half-kilometer radiance data to OCO-2: (i) for each OCO-2 sounding, the closest MODIS pixel is located using the matched MODIS pixel indices stored in OCO-2/MYD02HKM; (ii) Calibrated DN (SI-scaled Integer), radiance_scales, and radiance_offsets in MODIS Band 1 and Band 2 EV_250_Aggr500_RefSB data and Band 6 and 7 EV_500_RefSB data, are extracted for pixels that are inside a matchup box of $7 \times 7$ MODIS pixels centered at the closest MODIS pixel and are less than $2 \mathrm{~km}$ away from the center of the OCO-2 sounding; (iii) the MODIS DN data extracted in Step (ii) are converted to radiance in $W^{-2} \mathrm{sr}^{-1} \mu \mathrm{m}^{-1}$ using Equation (4) and the mean radiance value is taken as the matched MODIS radiance:

$$
L=\text { radiance_scales }(D N-\text { radiance_offsets })
$$

(5) Obtaining clear-sky OCO-2 soundings using cloud filtering (L2ABP + L2IDP + MODIS Cloud). We refer the readers to Ref. [15] for more details on cloud screening using OCO-2 L2ABP and L2IDP products.

(6) Constructing time series of OCO-2/MODIS radiance ratio for three OCO-2 bands using clear-sky soundings obtained in Step (5): (i) Extract OCO-2 Band 1, 2 and 3 continuum radiances from signal_o2_fph, signal_weak_co2_fph, signal_strong_co2_fph fields from OCO-2 L2Dia data structure; (ii) on a sounding-by-sounding basis, compute the ratio of OCO-2 Band 1 radiance to MODIS Band 1 and Band 2 radiance average, radiance ratio of OCO-2 Band 2 to MODIS Band 6, and radiance ratio of OCO-2 Band2 to MODIS Band 7; (iii) for each of the three OCO-2 bands, group the radiance ratios obtained in Step (ii) by the sensor overpass date, and plot mean and stddev radiance ratio of each overpass to assess the OCO-2 radiometric calibration stability. 
Define a ROI as a $0.9^{\circ} \times 0.9^{\circ}$ latitude by longitude area around the PICS center

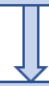

Extract OCO-2 L2ABP, L2IDP and L2Dia soundings in nadir and over ROI

Extract MODIS 1-km cloud data from OCO-2/MYD061KM and assign a clear or not clear flag for each OCO-2 sounding based on $7 \times 7$ MODIS pixels around the OCO-2 center lat/lon

Extract MODIS half-km radiance data from OCO2/MYD02HKM and collocate to OCO-2 sounding-by-sounding using a circular region with a $2 \mathrm{~km}$ radius around $0 \mathrm{CO}-2$ footprint

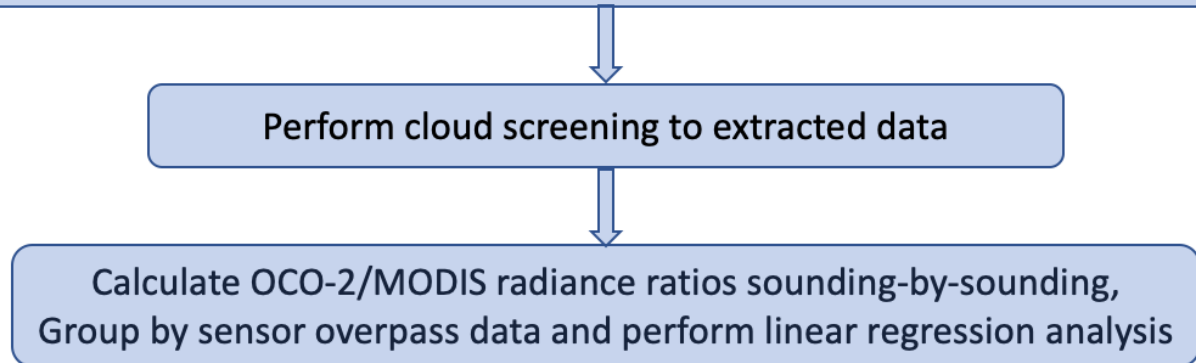

Figure 3. Schematic flow diagram for for OCO-2 and MODIS inter-comparision data processing for each of the eight PICS.

Note that our methodology does not consider cloud adjacency. However, for cloud screen we choose a box of $7 \times 7$ MODIS 1-km pixels around the OCO-2 lat/lon center point, and we have made the effective MODIS "footprint" much larger than the $\sim 1.5 \mathrm{~km} \times 2.5 \mathrm{~km}$ OCO-2 footprint. This ensures a very conservative "clear-sky" comparison in the sense that if any of those 49 MODIS pixels are probably cloudy we flag the entire scene as cloudy. Also note that for calculating the matched MODIS radiance for each OCO-2 sounding, we first choose a box of $7 \times 7$ MODIS half-km pixels around the OCO-2 lat/lon center point, then down select those pixels which are inside this box and are less than $2 \mathrm{~km}$ away from the OCO-2 lat/lon center point, and then take the mean radiance value of the down-selected pixels as the matached MODIS radiance.

\section{Results}

The left panel of Figure 4 shows the location of an example desert, Egypt1, $\left(27.12^{\circ} \mathrm{N}, 26.10^{\circ} \mathrm{E}\right)$ and its region-of-interest of a $0.9^{\circ} \times 0.9^{\circ}$ latitude by longitude area centered at the center. The OCO-2 swath is about $5 \mathrm{~km}$ wide, and is narrow in longitude compared to the ROI. The right panel shows the Band 1 continuum radiance map of obtained clear-sky OCO-2 nadir soundings over the Egypt1 site on 13 July 2019 (OCO-2 orbit 26754). The continuum radiances are normalized and are found to vary from $96 \%$ to $100 \%$ of the largest OCO-2 radiance within this ROI. This OCO-2 overpass is in the middle of the ROI and lasts about $18 \mathrm{~s}$, resulting in a total of about $400 \mathrm{OCO}-2$ soundings for this overpass. The number of clear-sky soundings varies from site to site and ranges from 50-400 soundings, depending on whether the overpass is in the middle of the ROI or in the corner. The nearly simultaneous MODIS observations with sensor viewing zenith angle of $\sim 15^{\circ}$ are mapped into the OCO-2 footprints using a circular region with a radius of $2 \mathrm{~km}$ around each OCO-2 footprint; and the OCO-2/MODIS radiance ratio is calculated sounding by sounding. 


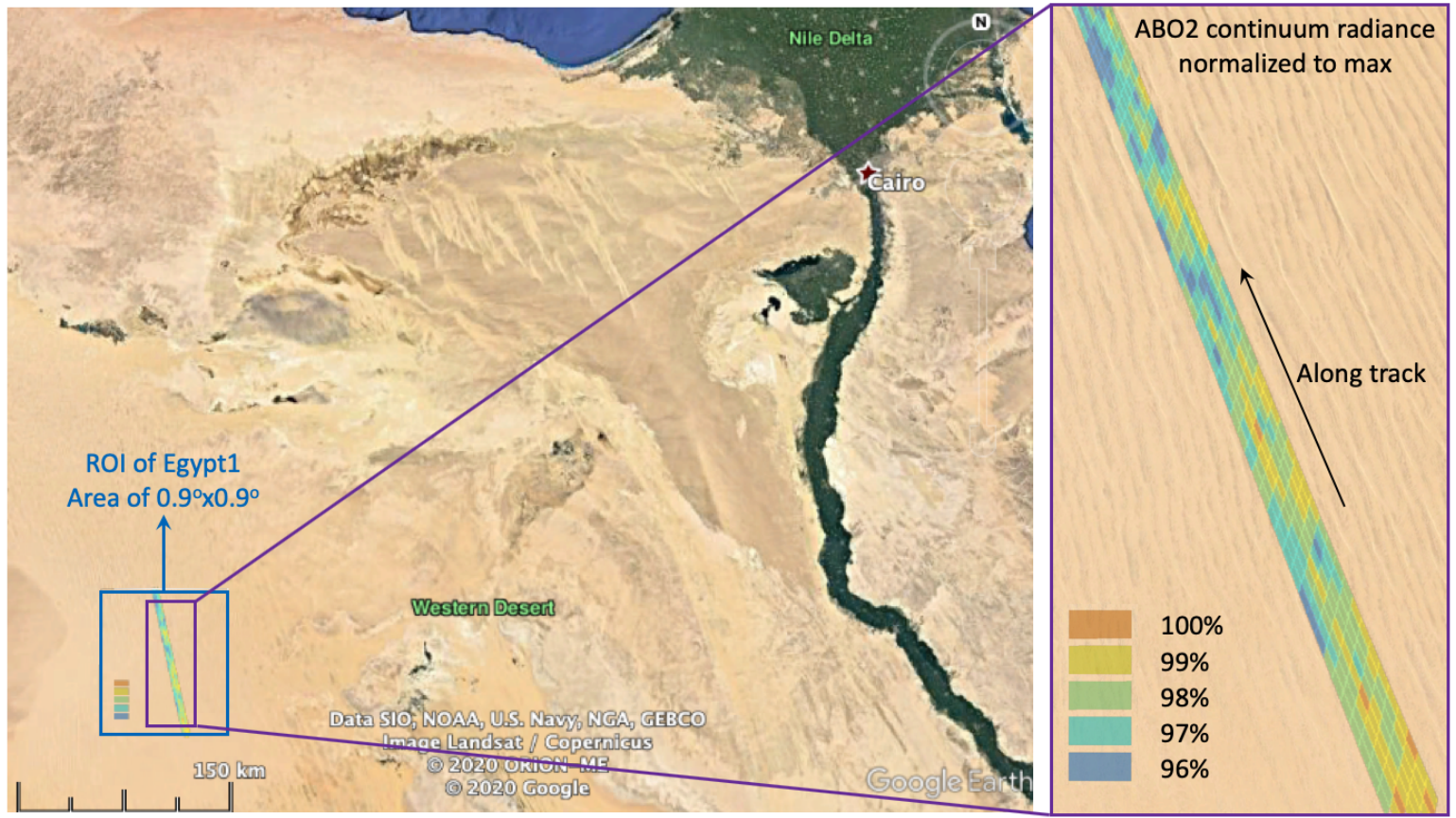

Figure 4. Location of the Egypt1 site and Band 1 continuum radiance map of $\sim 400$ clear-sky OCO-2 nadir soundings for one nadir overpass over the region-of-interest on 13 July 2019. MODIS swath is not shown and is significantly wider $(\sim 2330 \mathrm{~km})$.

Table 4 shows the median time of each OCO-2's clear-sky nadir scenes over Egypt1, together with the corresponding solar and MODIS viewing zenith and azimuth angles. It turns out that the VZA of the matched MODIS observations are $15 \pm 0.5^{\circ}$ for this site and all other seven sites. Note that a VZA window is not used as a criterion to select matched MODIS pixels. The three criteria used to select matched MODIS pixels are: (a) each OCO-2 science granule's observation times (typically $45 \mathrm{~min}$ ) are used to select the corresponding MODIS granules; (b) a filter is applied to MODIS granules selected in Step (a) to retain MODIS data within $\pm 50 \mathrm{~km}$ of the OCO-2 ground targets; (c) mapping MODIS radiance data down-selected in Step (b) to OCO-2 footprints. The root cause for the $\sim 15^{\circ}$ MODIS VZA is that the MODIS ground track is about $190 \mathrm{~km}$ West of the OCO-2 track; when MODIS views a ground target about $190 \mathrm{~km}$ away from its ground track at a height of $705 \mathrm{~km}$, the VZA is $\arctan (190 / 705) \approx 15^{\circ}$. Contributions to the variation in VZA include (1) temporal variation in the distance between MODIS and OCO-2 ground tracks and (2) $\sim 10 \mathrm{~km}$ difference among distances between the MODIS ground track and each of OCO-2 footprints due to OCO-2's $\sim 10 \mathrm{~km}$ swath.

Figure 5 presents the five-year time series of OCO-2/MODIS radiance ratio for all three OCO-2 bands over the Egypt1 site. Each data point represents one overpass: the $\mathrm{X}$ value is the median time of each overpass; the $Y$ value and its error bar are the mean and standard deviation of radiance ratio over all clear-sky soundings as shown in Figure 4. The blue dots in the top panel represent the result for the OCO-2 band 1, showing a downward trend with seasonal oscillations in Year 1 to Year 3, and showing an upward trend starting in Year 3. This "hook-shaped" trend is not seen in the other two bands over the same site, as shown in the middle and bottom panels. However, this hook in OCO-2 Band 1 is also clearly seen in other deserts such as Arabia1, Libya2 and Algeria4, as shown in Figure 6. 
Table 4. Median UTC times of OCO-2 clear-sky nadir passes over Egypt1 during the first five years of mission, together with the corresponding solar and MODIS zenith and azimuth angles.

\begin{tabular}{|c|c|c|c|c|c|c|c|c|}
\hline Year & Mon & Day & $\mathbf{H}$ & Min & Sol Zen & Sol Azi & MODIS Zen & MODIS Azi \\
\hline 2014 & 10 & 3 & 11 & 34 & 38.95 & -139.46 & 15.32 & -98.98 \\
\hline 2014 & 10 & 19 & 11 & 34 & 44.47 & -143.23 & 15.32 & -98.99 \\
\hline 2014 & 11 & 20 & 11 & 33 & 52.78 & -149.93 & 15.41 & -98.99 \\
\hline 2015 & 2 & 24 & 11 & 34 & 40.65 & -151.68 & 14.96 & -99.06 \\
\hline 2015 & 5 & 15 & 11 & 34 & 22.29 & -106.92 & 15.32 & -98.96 \\
\hline 2015 & 6 & 16 & 11 & 34 & 19.74 & -96.21 & 15.59 & -98.96 \\
\hline 2015 & 8 & 3 & 11 & 34 & 20.71 & -113.36 & 14.86 & -99.01 \\
\hline 2015 & 9 & 4 & 11 & 34 & 28.59 & -130.21 & 14.77 & -99.02 \\
\hline 2015 & 11 & 7 & 11 & 33 & 49.95 & -147.35 & 15.23 & -98.99 \\
\hline 2015 & 12 & 9 & 11 & 33 & 54.75 & -153.43 & 15.14 & -99.00 \\
\hline 2016 & 1 & 10 & 11 & 34 & 52.65 & -157.02 & 14.96 & -99.03 \\
\hline 2016 & 2 & 11 & 11 & 34 & 44.74 & -155.04 & 14.69 & -99.07 \\
\hline 2016 & 3 & 14 & 11 & 34 & 34.73 & -144.69 & 15.32 & -99.00 \\
\hline 2016 & 4 & 15 & 11 & 34 & 26.62 & -125.80 & 16.41 & -98.88 \\
\hline 2016 & 6 & 18 & 11 & 33 & 19.39 & -96.38 & 14.96 & -99.03 \\
\hline 2016 & 7 & 20 & 11 & 33 & 19.17 & -105.81 & 14.68 & -99.04 \\
\hline 2016 & 8 & 21 & 11 & 33 & 24.57 & -124.33 & 14.78 & -99.05 \\
\hline 2016 & 9 & 22 & 11 & 33 & 35.04 & -136.84 & 14.69 & -99.05 \\
\hline 2016 & 10 & 24 & 11 & 33 & 46.17 & -144.67 & 14.96 & -99.03 \\
\hline 2016 & 11 & 25 & 11 & 33 & 53.51 & -151.15 & 15.05 & -99.02 \\
\hline 2016 & 12 & 27 & 11 & 33 & 54.30 & -156.09 & 14.87 & -99.04 \\
\hline 2017 & 3 & 1 & 11 & 34 & 38.85 & -150.16 & 14.96 & -99.05 \\
\hline 2017 & 4 & 2 & 11 & 34 & 29.54 & -134.62 & 15.59 & -98.95 \\
\hline 2017 & 6 & 5 & 11 & 34 & 20.22 & -98.22 & 15.05 & -99.01 \\
\hline 2017 & 7 & 7 & 11 & 33 & 18.78 & -99.64 & 14.77 & -99.03 \\
\hline 2017 & 11 & 12 & 11 & 33 & 51.17 & -148.49 & 14.96 & -99.02 \\
\hline 2017 & 12 & 14 & 11 & 34 & 54.89 & -154.31 & 14.78 & -99.05 \\
\hline 2018 & 1 & 15 & 11 & 34 & 51.65 & -157.10 & 14.87 & -99.06 \\
\hline 2018 & 3 & 20 & 11 & 35 & 33.15 & -141.91 & 14.95 & -99.02 \\
\hline 2018 & 6 & 24 & 11 & 34 & 19.22 & -96.60 & 14.77 & -99.04 \\
\hline 2018 & 7 & 26 & 11 & 34 & 19.86 & -108.70 & 15.77 & -98.95 \\
\hline 2018 & 8 & 27 & 11 & 34 & 26.29 & -126.54 & 15.87 & -98.92 \\
\hline 2018 & 9 & 28 & 11 & 33 & 37.09 & -138.10 & 15.05 & -99.00 \\
\hline 2018 & 10 & 30 & 11 & 34 & 47.83 & -145.68 & 15.05 & -99.02 \\
\hline 2019 & 2 & 3 & 11 & 34 & 46.96 & -156.11 & 14.86 & -99.04 \\
\hline 2019 & $\overline{4}$ & 8 & 11 & 34 & 28.17 & -131.03 & 15.14 & -98.99 \\
\hline 2019 & 5 & 10 & 11 & 34 & 22.69 & -110.10 & 14.87 & -99.03 \\
\hline 2019 & 6 & 11 & 11 & 34 & 19.85 & -97.07 & 14.77 & -99.03 \\
\hline \multirow[t]{3}{*}{2019} & 7 & 13 & 11 & 34 & 18.87 & -101.80 & 14.68 & -99.03 \\
\hline & & & & mean & 35.10 & -131.43 & 15.08 & -99.01 \\
\hline & & & & stddev & 13.22 & 21.52 & 0.37 & 0.04 \\
\hline
\end{tabular}

The hook-shaped trend seen in the OCO-2 Band 1 radiance ratio indicates that the corresponding radiometric calibration of the OCO-2 Build 8R data product changed starting Year 3.2. This is the result of incorrectly modeling solar calibrator aging for the Band 1 spectrometer. During routine operations, ice buildup on the OCO-2 FPAs reduces the instrument throughput. To address this problem, the OCO-2 FPAs and optical bench are warmed to either 12 or $28 \mathrm{C}$ on semi-annual to annual time scales to decontaminate the FPAs and restore the sensitivity of the instrument (details in Ref. [1]). The twelve black dots in Figure 7 denote the solar observations recorded immediately after each decontamination cycle when there was no icing on the FPAs. At these times, the Build 8 L1B products assumed that any deviations in the solar calibration observations seen collected early in the mission were due to aging of the solar calibrator. This aging was removed with a fit of these decontamination-cycle-start solar measurements vs time with a simple exponential function. After 2.5 years in orbit, this function was extrapolated to estimate the solar calibrator degradation, 
but this underestimated the actual throughput degradation. The red trace in Figure 7 denotes the model that incorrectly uses the exponential fit to the first eight points instead of linearly interpolating between all twelve points. Underestimating solar calibrator aging leads to a larger value for the calibrated radiance of the latest data after gain degradation coefficients are applied. After correcting for this error, the data after Year 3.2 follow the trend from previous years, as shown by orange points in Figures 5 and 6.

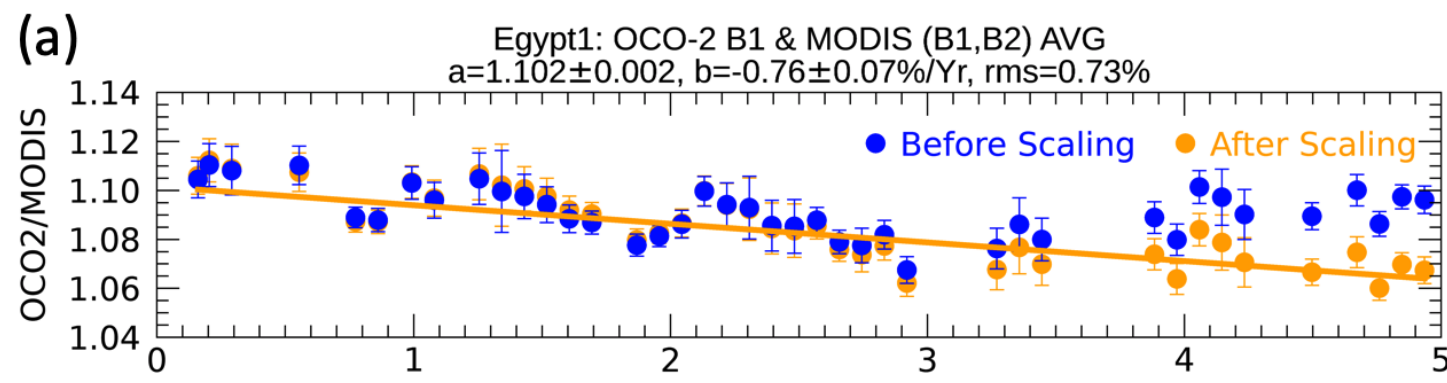

(b)

Egypt1: OCO-2 B2 \& MODIS B6

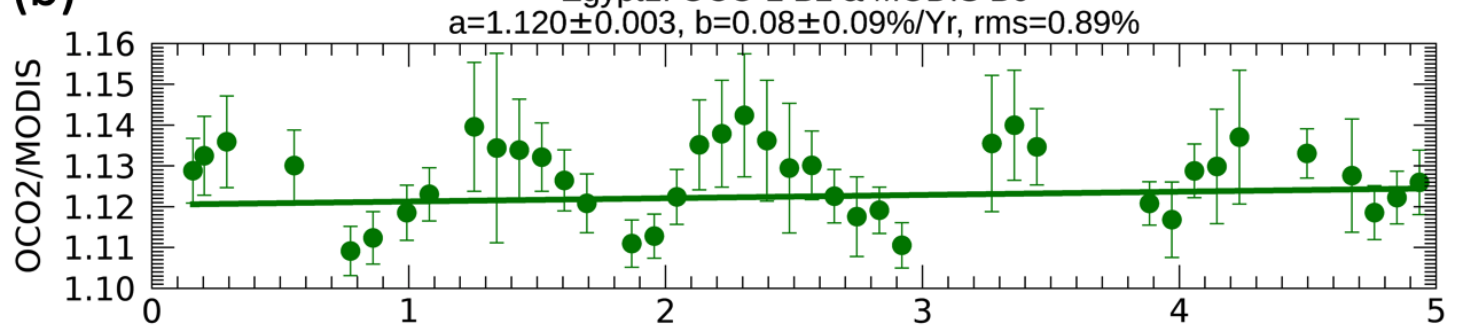

(c)

Egypt1: OCO-2 B3 \& MODIS B7

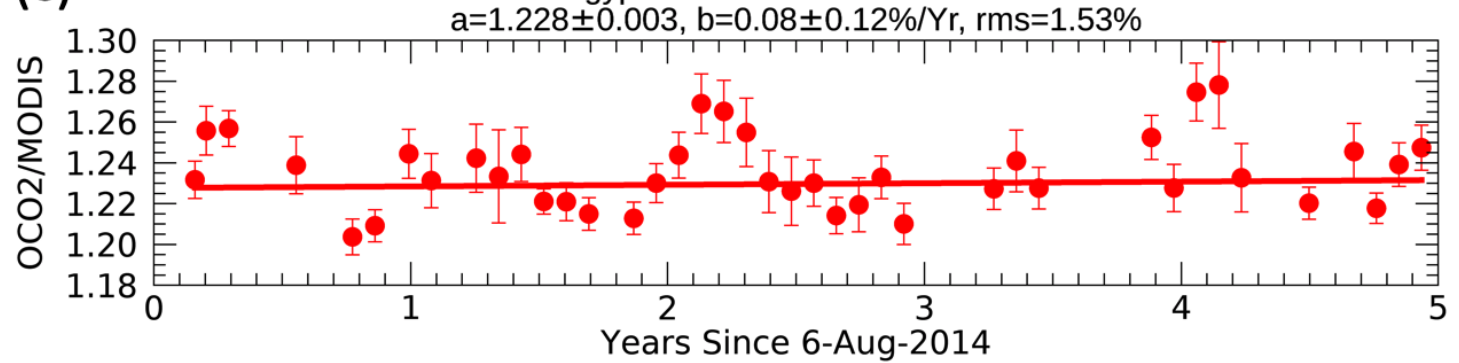

Figure 5. Five-year time seriesof the OCO-2/MODIS radiance ratio for all three OCO-2 bands over Egypt1: Panel (a) for OCO-2 Band 1; Panel (b) for OCO-2 Band 2; Panel (c) for OCO-2 Band 3. In Panel (a), Orange data points denote the result corrected for an error in modeling the aging of the solar calibrator.

Figures 5 and 6 also show the linear regression of the OCO-2/MODIS radiance ratio with the solar calibration error corrected for Band 1 (orange points), with the slopes in \% per year and the intercept listed in the title of each panel. Table 5 summarizes these linear regression results as well as those for Band 2 and 3 as shown in Figures A1 and A2 (Appendix A). During the first five years, the Egypt1 site has the most clear-sky nadir overpasses, 39, among the eight Desert sites, followed by Arabia1, Libya2 and Algeria4 with 35, 32 and 29 points, respectively. The other four sites have less than 20 data points. Note that during a five-year period, a maximum of 57 nadir overpasses for each site is possible, because the OCO-2 orbit repeats every 16 days and OCO-2 observes in nadir mode for only half of these paths. 

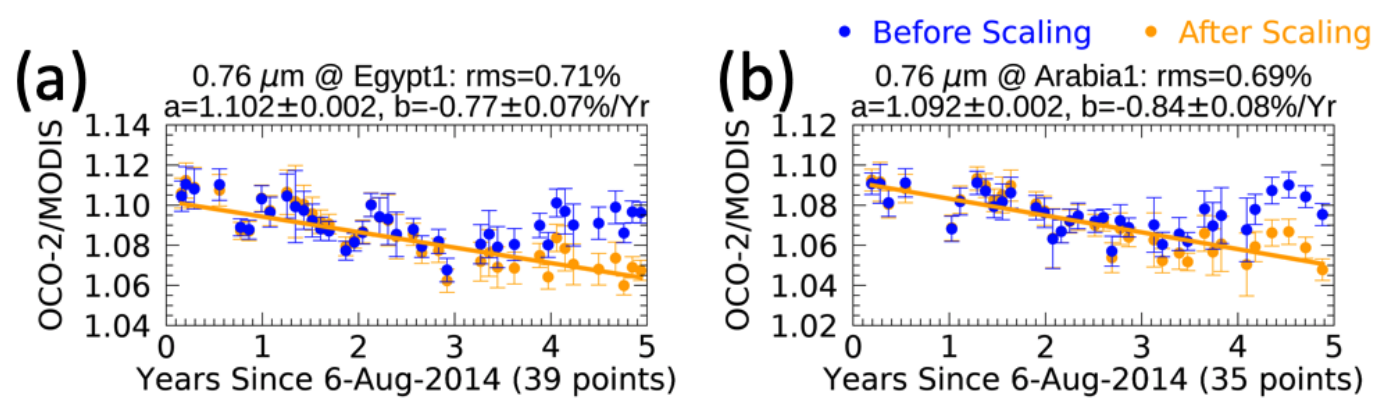

(c)

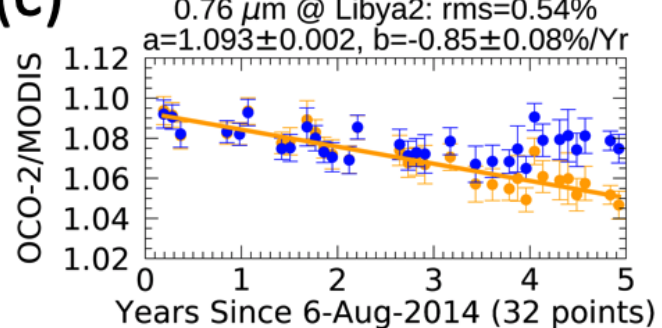

(e)

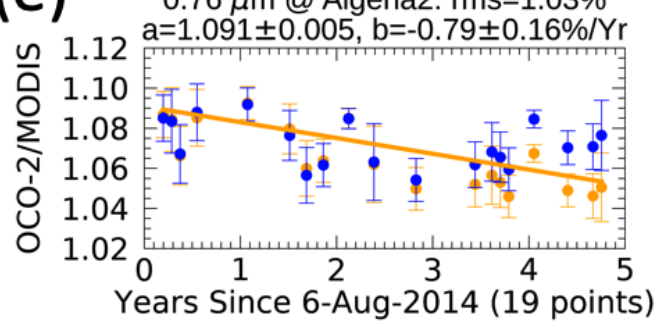

(g)

$0.76 \mu \mathrm{m} @$ Algeria1: rms $=0.78 \%$

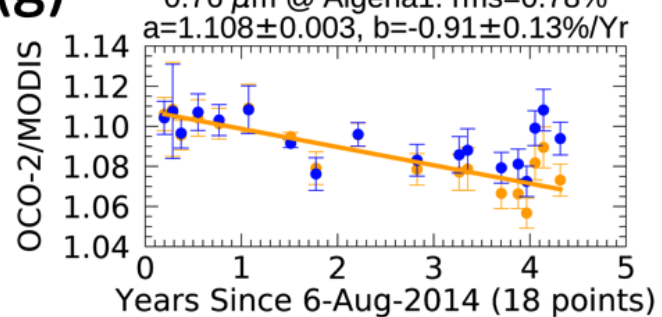

(d) $\quad 0.76 \mu \mathrm{m}$ @ Algeria4: rms $=0.89 \%$

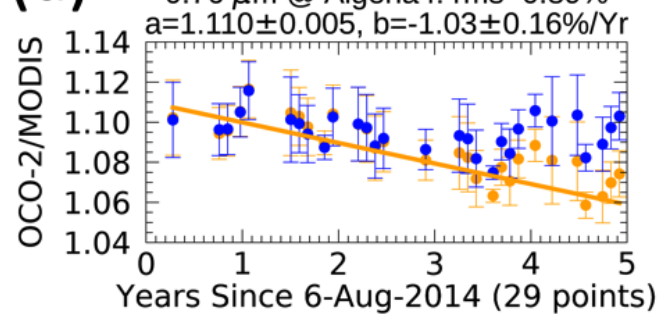

(f)

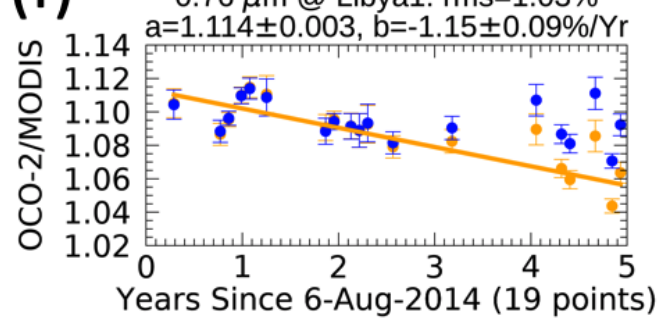

(h) $0.76 \mu \mathrm{m} @$ Mauritania1: $\mathrm{rms}=0.76 \%$

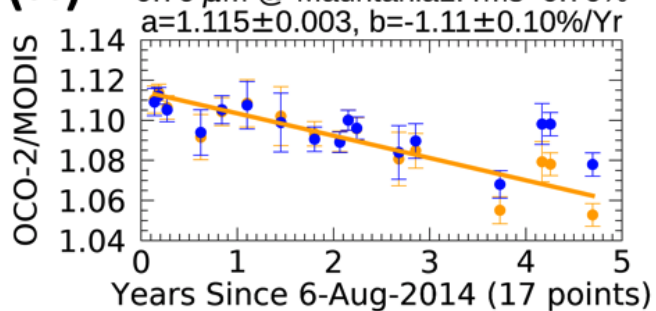

Figure 6. Five-year time series of the OCO-2/MODIS radiance ratio for OCO-2 Band 1 over eight desert sites: Panel (a) is over Egypt1; Panel (b) over Arabia1; Panel (c) over Libya2; Panel (d) over Algeria4; Panel (e) over Algeria2; Panel (f) over Libya1; Panel (g) over Algeria1; Panel (h) over Mauritania1. Orange data points denote the result corrected for an error on modeling the aging of the solar calibrator. 


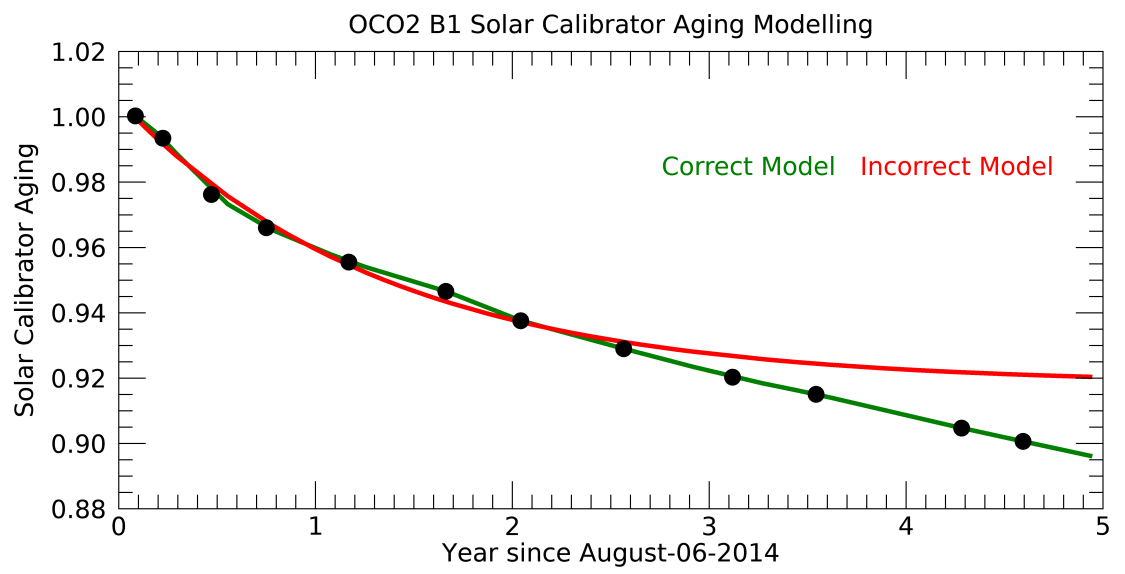

Figure 7. Correcting the aging of the solar calibrator: (red) model that incorrectly uses the exponential fit to the first eight points instead of the one to all twelve points; (green) correct model.

Table 5. Summary of the slope and intercept for linear regression of OCO-2/MODIS radiance ratio. The second last column is the number of clear-sky OCO-2 passes over each site within the first five-year OCO-2 mission. The last column is the average number of clear soundings for each overpass.

\begin{tabular}{|c|c|c|c|c|c|c|c|c|}
\hline \multirow[b]{2}{*}{ Site } & \multicolumn{3}{|c|}{ Slope (\% per Year) } & \multicolumn{3}{|c|}{ Intercept } & \multirow[b]{2}{*}{ Passes } & \multirow[b]{2}{*}{ Sndngs } \\
\hline & Band 1 & Band 2 & Band 3 & Band 1 & Band 2 & Band 3 & & \\
\hline Egypt1 & $-0.77 \pm 0.07$ & $0.08 \pm 0.09$ & $0.08 \pm 0.12$ & $1.102 \pm 0.002$ & $1.120 \pm 0.003$ & $1.228 \pm 0.003$ & 39 & 216 \\
\hline Libya2 & $-0.85 \pm 0.08$ & $0.04 \pm 0.08$ & $-0.32 \pm 0.11$ & $1.093 \pm 0.002$ & $1.124 \pm 0.002$ & $1.239 \pm 0.003$ & 32 & 155 \\
\hline Algeria4 & $-1.03 \pm 0.16$ & $0.14 \pm 0.13$ & $-0.58 \pm 0.18$ & $1.110 \pm 0.005$ & $1.112 \pm 0.004$ & $1.234 \pm 0.006$ & 29 & 125 \\
\hline Algeria2 & $-0.79 \pm 0.16$ & $0.05 \pm 0.15$ & $-0.17 \pm 0.19$ & $1.091 \pm 0.005$ & $1.117 \pm 0.004$ & $1.225 \pm 0.006$ & 19 & 135 \\
\hline Libya1 & $-1.15 \pm 0.09$ & $0.11 \pm 0.07$ & $-0.24 \pm 0.12$ & $1.114 \pm 0.003$ & $1.127 \pm 0.003$ & $1.246 \pm 0.004$ & 19 & 144 \\
\hline mean(first 3) & -0.82 & 0.08 & -0.18 & 1.096 & 1.118 & 1.235 & & \\
\hline stddev(first 3) & 0.04 & 0.05 & 0.22 & 0.006 & 0.007 & 0.006 & & \\
\hline mean(all 8) & -0.93 & 0.06 & -0.35 & 1.103 & 1.120 & 1.233 & & \\
\hline stddev(all 8) & 0.15 & 0.06 & 0.26 & 0.010 & 0.007 & 0.016 & & \\
\hline
\end{tabular}

\section{Discussion}

As shown in Table 5 for OCO-2 Band 1 at $0.765 \mu \mathrm{m}$, the slopes of the fitted regression lines for the three sites with more than 30 data points, Egypt1, Arabia1 and Libya2, agree very well with each other, within $1 \sigma$ error. The mean and standard deviation of these slopes over these three sites are $-0.82 \%$ per year and $0.04 \%$ per year. For Algeria 4 with 29 data points, the error bar for each individual data point, which is the standard deviation of radiance ratio over all clear-sky soundings for each overpass, is typically twice as large than that for the above three sites. This result agrees with the reported relatively large spatial inhomogeneity of Algeria4 (see Table III of Ref. [12]). As a result, the determined slope error bar, $0.16 \%$, is twice as large as those for the above three sites; the fit rms, $0.89 \%$, is also worse. The slopes for the other four sites with less than 20 data points are also determined with similar error bars as Algeria4, and with large discrepancies. The large scatter in the determined slopes for these four sites may be attributed to data with seasonal oscillations, especially with few complete periods. The slopes derived from such fits can be biased by: (1) data points not starting and ending exactly at the same phase; (2) inhomogeneous sampling and under-sampling of the seasonal cycle.

The radiometric calibration drifts in the OCO-2 Band 2 at 1.61 micron and Band 3 at 2.06 micron are much smaller than that in Band 1 at 0.765 micron. Figure A1 shows that for Band 2, the slow slope for each of the eight sites has an uncertainty larger than its value. The mean and standard deviation over all eight desert sites are $0.06 \%$ per year and $0.08 \%$ per year, similar to those over the three sites with more than 30 data points. This indicates a drift of less than $0.1 \%$ per year in the OCO-2 Band 2 radiometric calibration. 
In Figure A2 for the OCO-2 Band 3 at $2.06 \mu \mathrm{m}$, the slopes for the eight sites vary from $-0.58 \%$ per year to $0.75 \%$ per year. The mean and standard deviation over all eight desert sites are $-0.35 \%$ per year and $0.26 \%$ per year, compared to $-0.18 \%$ per year and $0.22 \%$ per year over the three sites with more than 30 data points. This indicates a drift of less than $0.3 \%$ per year in the OCO-2 Band 3 radiometric calibration. The scatter of radiance ratio within a single site in Band 3 is twice as large as in the other two bands. At least two factors have been identified to explain the large discrepancy among slopes from different sites and the larger scatter of radiance ratio within a single site. First, unlike in Band 1 and Band 2, there is absorption throughout Band 3, so the true continuum level is out of band and the "continuum" level estimated by the largest 10 columns' radiance is biased. This bias is coupled to XCO2, Surface reflectance and atmosphere temperature, which change with time. Second, this is the longest wavelength among the three OCO-2 bands and both OCO-2 Band 3 and MODIS Band 7 are most sensitive to thermal emission from Earth's surface.

The estimated radiometric drift for the OCO-2 Band $1,-0.82 \pm 0.04 \%$ per year over the three sites with more than 30 overpasses, is consistent with the $-0.6 \pm 0.2 \%$ per year trend derived using OCO-2 lunar observations. This trend is attributed to the OCO-2 Band 1 instrument degradation. Supported by the lunar trend and the OCO-2/MODIS trend, an upward correction of $0.6 \%$ per year has been adopted for OCO-2 Band 1 in the next OCO-2 version, Build 10R. The estimated drifts of $0.08 \pm 0.05 \%$ per year and $-0.18 \pm 0.22 \%$ per year for the Band 2 and band 3 largely agree with the $-0.2 \pm 0.2 \%$ per year and $-0.2 \pm 0.2 \%$ per year trend derived using the lunar observations.

The seasonal oscillations in the OCO-2/MODIS radiance ratio are most obviously seen in the OCO-2 Band 2 and Band 3 (See Figures 5, A1 and A2). These oscillations mainly arise from the $\sim 15^{\circ}$ viewing zenith angle difference between OCO-2 and MODIS: the seasonal variation of the surface Bidirectional Reflectance Distribution Function (BRDF) at nadir is different from that at $15^{\circ}$. In a follow-up paper, we will improve the accuracy of the slopes by normalizing the seasonal variations using a semi-empirical BRDF model. Another minor contributor to the seasonal oscillations in the OCO-2/MODIS radiance ratio may be the uncorrected effects of polarized signals from the desert scenes. The degree of polarization and the angle of the linear polarization are the two necessary parameters in the polarization distribution models that are needed in correcting the polarization effects. Ref. [16] has modeled these two quantities for ocean scenes, and Ref. [17] for different scene types including desert. As shown in Table 4, the OCO-2 observations used in the current study are acquired at nadir over desert with solar zenith angle ranging from $18^{\circ}$ to $55^{\circ}$ and solar azimuth angle varying from about $-100^{\circ}$ to $-160^{\circ}$, leading to $1 \%$ to $3 \%$ polarization of the reflected solar radiance based on results from Ref. [17]. OCO-2 collects observations with the normal to the long axis of the spectrometer slits rotated 30 degrees (clockwise from above) from the principal plane, defined by the sun, surface footprint and instrument aperture [1]. At this orientation, the range of solar illumination angles could introduce a $\sim 1 \%$ temporal temporal variation in the degree of polarization of the reflected sunlight observed by OCO-2.

The mean intercepts over all eight desert sites, 1.103, 1.120 and 1.233 for the three OCO-2 bands, are comparable to their counterparts over the three desert sites with more 30 data points, 1.096, 1.118, 1.235. The standard deviations are on the order of 0.01 . In theory, the OCO-2 absolute radiometric bias can be estimated from the intercept value, after accounting for the following two factors in the intercept: (1) mismatched relative spectral response functions of OCO-2 and MODIS bands as shown in Figure 2; (2) viewing geometry differences, which can be corrected with the bidirectional reflectance distribution function. For each inter-comparison data pair, the OCO-2 sensor viewing geometry is nadir while the MODIS sensor viewing zenith angle is about $15^{\circ}$. In a follow-up paper, we will quantify these effects with mature methodologies existing in literature (see Ref. [18] and references therein). 
The methodology employed in the current study, comparing OCO-2 and MODIS radiances using OCO-2 nadir observations and nearly simultaneous MODIS observations with sensor viewing zenith angles of $15^{\circ}$, effectively quantified a drift in OCO-2 Build 8 radiometric calibration and captured a small error in correcting OCO-2's solar calibrator aging. This methodology provides an alternate means to compare two sensors such as OCO-2 and MODIS which can not be compared using the widely used simultaneous nadir overpass (SNO) methodology. SNOs do not occur between OCO-2 and Aqua MODIS because their ground tracks are nearly parallel and have a large distance between them.

The cross calibration of OCO-3 with other sensors can be performed with the traditional SNO methodology, which is limited to nadir observations. The methodology presented in this study can also be applied to cross calibration of OCO-3. The ISS orbit in OCO-3 will change the matchup pattern, leading to less matchups compared to cross calibration of OCO-2 with MODIS. A MODIS VZA window can be used as one of the criteria to allow MODIS observations at multiple VZA to be included, thus increasing matchups. Then normalizing the multi-VZA MODIS observations to a common VZA is needed before trending the radiance ratio of OCO-3 to a reference sensor.

\section{Conclusions}

This paper has quantified the OCO-2 radiometric calibration stability using OCO-2 nadir observations over eight desert sites and nearly simultaneous Aqua MODIS observations with sensor viewing zenith angles of $15 \pm 0.5^{\circ}$. The MODIS data are collocated into the OCO-2 geolocation grid using a circular region with a radius of $2 \mathrm{~km}$ around OCO-2 footprint. For each of the three OCO-2 bands, the radiance at the continuum level is compared to the in band spectral radiance of the matching MODIS band. A hook-shaped trend is seen in the OCO-2/MODIS radiance ratio for OCO-2 Band 1, and the root cause is attributed to a small error in modeling the OCO-2 solar calibration for this band. This error has been corrected in the next OCO-2 version, Build 10R. No hook-shaped trend is seen for OCO-2 Bands 2 or 3.

A drift of $-0.8 \pm 0.1 \%$ per year in the OCO-2 Band 1 is revealed by linear regressions of the OCO-2/MODIS radiance ratio over three desert sites with more than 30 data points over a five-year period. For OCO-2 Bands 2 and 3, the trend is determined with an uncertainty larger than its value, indicating less than $0.1 \%$ and less than $0.3 \%$ per year drift in the radiometric calibration of Band 2 and Band 3, respectively. These trending results are consistent with the ones derived using the lunar observation, $-0.6 \pm 0.2 \%,-0.2 \pm 0.2 \%,-0.2 \pm 0.2 \%$ per year, for the three bands, respectively. An upward correction of $0.6 \%$ per year is performed for OCO-2 Band 1 in in the next OCO-2 version, Build 10R.

In a follow-up paper, we will examine OCO-2's absolute radiometric calibration embedded in the intercepts for the linear regressions performed in this study, by developing BRDF models to account for the viewing geometry difference and to reduce the seasonal oscillations seen in radiance ratio, and by computing the expected bias due to mismatching relative response functions.

Author Contributions: Methodology, S.Y., A.E.; software, S.Y., T.T., L.C., D.F., R.L.; formal analysis, S.Y., R.R., C.B., A.A.; data curation, H.C.; writing-original draft preparation, S.Y., R.R.; writing-review and editing, R.R., T.T., A.A., C.B.; supervision, A.E., D.C., C.O., X.X. All authors have read and agreed to the published version of the manuscript.

Funding: Part of the research described in this paper was carried out at the Jet Propulsion Laboratory, California Institute of Technology, under a contract with the National Aeronautics and Space Administration (80NM0018D0004). The CSU contribution to this work was supported by JPL subcontract \#1439002. Government sponsorship is acknowledged. (c) 2020. All rights reserved.

Acknowledgments: We would like to thank Mark Richardson for discussion on retrieving the MODIS viewing geometry from the OCO-2/MODIS collocated data products. We thank Graziela Keller Rodrigues for discussion on biases associated with linear fits to data with seasonal oscillations and with few complete periods.

Conflicts of Interest: The authors declare no conflict of interest. 


\section{Abbreviations}

The following abbreviations are used in this manuscript:

$\begin{array}{ll}\text { RDF } & \text { Bidirectional Reflectance Distribution Function } \\ \text { FPA } & \text { Focal Plane Array } \\ \text { ILS } & \text { Instrument Line Shape } \\ \text { MODIS } & \text { Moderate Resolution Imaging Spectroradiometer } \\ \text { NASA } & \text { National Aeronautics and Space Administration } \\ \text { OCO-2 } & \text { Orbiting Carbon Observatory 2 } \\ \text { OCO-3 } & \text { Orbiting Carbon Observatory 3 } \\ \text { PICS } & \text { Pseudo Invariant Calibration Sites } \\ \text { RRV } & \text { Railroad Valley } \\ \text { RSR } & \text { Relative Spectral Response } \\ \text { TOA } & \text { Top of Atmosphere } \\ \text { XCO2 } & \text { column-average dry air mole fraction o fCO2 }\end{array}$

\section{Appendix A}
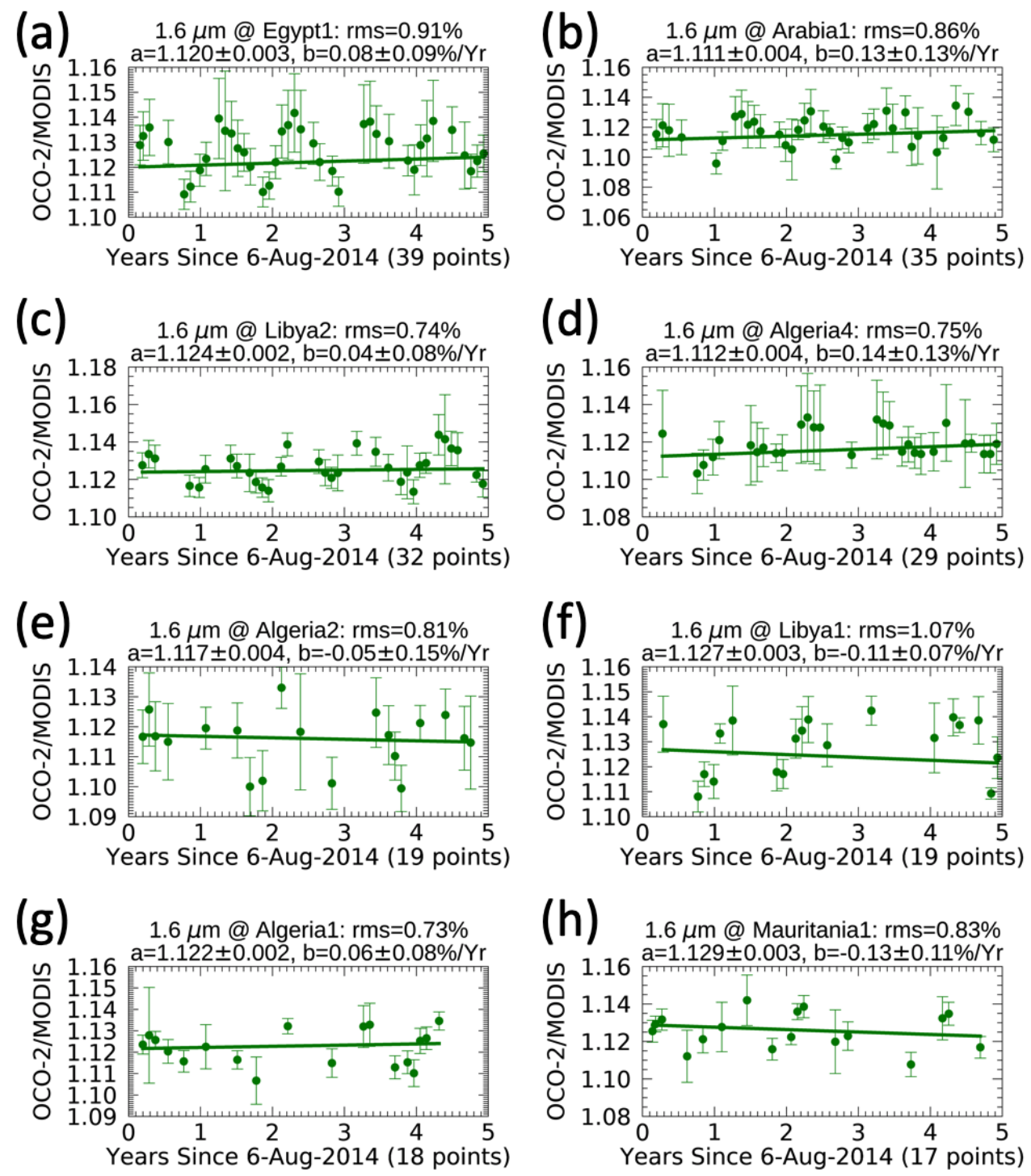

Figure A1. Five-year time series ofthe OCO-2/MODIS radiance ratio for OCO-2 Band 2 over eight desert sites: Panel (a) is over Egypt1; Panel (b) over Arabia1; Panel (c) over Libya2; Panel (d) over Algeria4; Panel (e) over Algeria2; Panel (f) over Libya1; Panel (g) over Algeria1; Panel (h) over Mauritania1. 

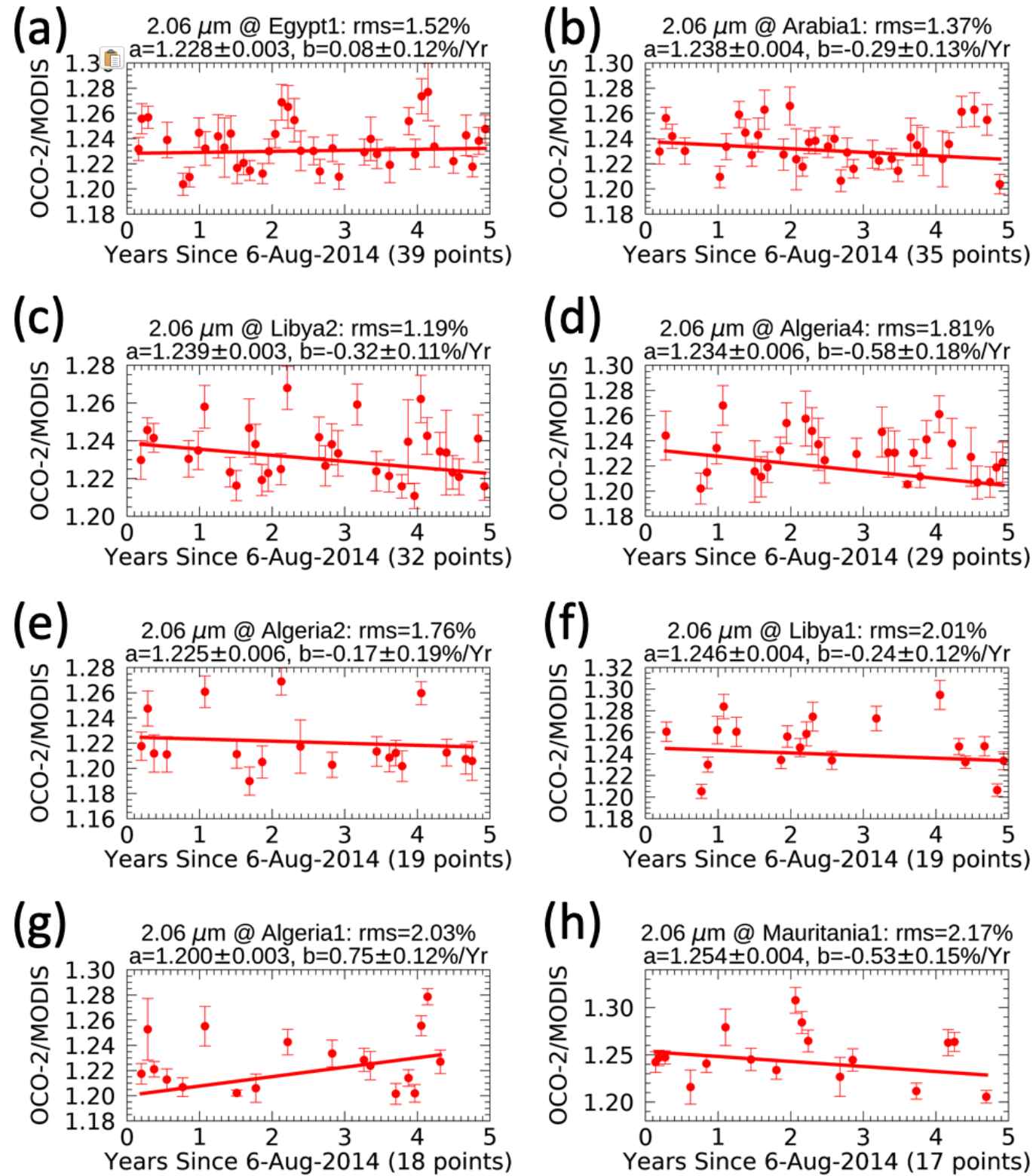

Figure A2. Five-year time series ofthe OCO-2/MODIS radiance ratio for OCO-2 Band 3 over eight desert sites: Panel (a) is over Egypt1; Panel (b) over Arabia1; Panel (c) over Libya2; Panel (d) over Algeria4; Panel (e) over Algeria2; Panel (f) over Libya1; Panel (g) over Algeria1; Panel (h) over Mauritania1.

\section{References}

1. Crisp, D.; Pollock, H.R.; Rosenberg, R.; Chapsky, L.; Lee, R.A.M.; Oyafuso, F.A.; Frankenberg, C.; O’Dell, C.W.; Bruegge, C.J.; Doran, G.B.; et al. The on-orbit performance of the Orbiting Carbon Observatory-2 (OCO-2) instrument and its radiometrically calibrated products. Atmos. Meas. Tech. 2017, 10, 59-81. [CrossRef]

2. Orbiting Carbon Observatory-3 (OCO-3) Project Web Site. Available online: https://oco3.jpl.nasa.gov/ (accessed on 5 April 2020).

3. Eldering, A.; Taylor, E.T.; O'Dell, C.W.; Pavlick, R. The OCO-3 mission: measurement objectives and expected performance based on 1 year of simulated data. Atmos. Meas. Tech. 2019, 12, 2341-2370. [CrossRef]

4. Eldering, A.; Bennett, M.; Cervantes, D.; Chang, A.; Cheng, C.; Crisp, D.; Gang, L.; FIsher, B.; Kiel, M.; Kurosu, T.; et al. OCO-3 early mission performance. In preparation.

5. Rosenberg, R.; Maxwell, S.; Johnson, B.C.; Chapsky, L.; Lee, R.; Pollock, R. Preflight Radiometric Calibration of Orbiting Carbon Observatory 2, IEEE Trans. Geosci. Remote Sens. 2013, 55, 1994-2006 [CrossRef] 
6. Bruegge, C.; Crisp, D.; Helmlinger, M.; Kataoka, F.; Kuze, A.; Lee, R.; McDuffie, J.; Rosenberg, R.; Schwandner, F.; Shiomi, K.; et al. Vicarious Calibration of Orbiting Carbon Observatory-2. IEEE Trans. Geosci. Remote Sens. 2019, 57, 5135-5145. [CrossRef]

7. Chander, G.; Hewison, T.; Fox, N.; Wu, X.; Xiong, X.; Blackwell, W.J. Overview of Intercalibration Of Satellite Instruments. IEEE Trans. Geosci. Remote Sens. 2013, 51, 1056-1080. [CrossRef]

8. Xiong, X.; Angal A.; Twedt, K.A.; Chen, H.; Link, D.; Geng, X.; Aldoretta, E.; Mu, Q. MODIS Reflective Solar Bands On-Orbit Calibration and Performance. IEEE Trans. Geosci. Remote Sens. 2019, 57, 6355-6371. [CrossRef]

9. Angal, A.; Xiong, X.; Wu, A.; Geng, X.; Chen, H. Improvements in the On-Orbit Response Versus Scan Angle Characterization of the Aqua MODIS Reflective Solar Bands. IEEE Trans. Geosci. Remote Sens. 2018, 56, 1728-1738. [CrossRef]

10. Lee, R.A.M.; O’Dell, C.; Wunch, D.; Roehl, C.M.; Osterman, G.B.; Blavier, J.-F.; Rosenberg, R.; Chapsky, L.; Frankenberg, C.; Hunyadi-Lay, S.L.; et al. Preflight Spectral Calibration of the Orbiting Carbon Observatory 2. IEEE Trans. Geosci. Remote Sens. 2017, 55, 2499-2508. [CrossRef]

11. Cosnefroy, H.; Leroy, M.; Briotte, X. Selection and characterization of Saharan and Arabian desert sites for the calibration of optical satellite sensors. Remote Sens. Environ. 1996, 58, 101-114. [CrossRef]

12. Lachérade, S.; Fougnie, B.; Henry, P.; Gamet, P. Cross-calibration over desert sites: Description, methodology and operational implementation. IEEE Trans. Geosci. Remote Sens. 2013, 51, 1098-1113. [CrossRef]

13. Bacour, C.; Briottet, X.; Breon, F.-M.; Viallefont-Robinet, F.; Bouvet, M. Revisiting Pseudo Invariant Calibration Sites (PICS) Over sand deserts for vicarious calibration of optical imagers at $20 \mathrm{~km}$ and $100 \mathrm{~km}$ Scales. Remote Sens. 2019, 11, 1166. [CrossRef]

14. Cronk, H. OCO-2/MODIS Collocation Products User Guide. Version 3. June 2018. Available online: ftp:/ /ftp.cira.colostate.edu/ftp/TTaylor/publications/ (accessed on 5 April 2020).

15. Taylor, T.E.; O’Dell, C.W.; Frankenberg, C.; Partain, P.; Cronk, H.Q.; Savtchenko, A.; Nelson, R.R.; Rosenthal, E.J.; Chang, A.Y.; Fisher, B.; et al. Orbiting Carbon Observatory-2 (OCO-2) cloud screening algorithms; validation against collocated MODIS and CALIOP data. Atmos. Meas. Tech. 2016, 9, 973-989. [CrossRef]

16. Sun, W.; Lukashin, C.; Baize, R.; Goldin, D. Modeling polarized solar radiation for CLARREO inter-calibration applications: Validation with PARASOL data. J. Quant. Spectrosc. Radiat. Transf. 2014, 150, 121-133. [CrossRef]

17. Nada, F.; Breon, F.-M. Parameterization of surface polarized reflectance derived from POLDER spaceborne measurements. IEEE Trans. Geosci. Remote Sens. 1999, 37, 1709-1718. [CrossRef]

18. Angal, A.; Xiong, X.; Wu, A.; Chander, G.; Choi, T. Multitemporal cross-calibration of the Terra MODIS and Landsat 7 ETM+ reflectance solar bands. IEEE Trans. Geosci. Remote Sens. 2017, 51, 1870-1882.

(C) 2020 by the authors. Licensee MDPI, Basel, Switzerland. This article is an open access article distributed under the terms and conditions of the Creative Commons Attribution (CC BY) license (http://creativecommons.org/licenses/by/4.0/). 